International Journal of Pure and Applied Mathematics

Volume 82 No. 4 2013, 581-593

ISSN: 1311-8080 (printed version); ISSN: 1314-3395 (on-line version)

url: http://www.ijpam.eu

doi: http://dx.doi.org/10.12732/ijpam.v82i4.7

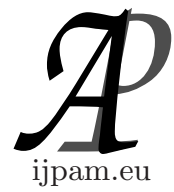

\title{
INVERSE ESTIMATION OF THE INITIAL CONDITION FOR THE HEAT EQUATION
}

\author{
Tao Min ${ }^{1}$, Bei Geng ${ }^{2}$, Jucheng Ren ${ }^{3}$ \\ Xi'an University of Technology \\ Xi'an Shaanxi, 710054, P.R. CHINA
}

\begin{abstract}
In this work, we investigate the inverse problem in the heat equation involving the recovery of the initial temperature from measurements of the final temperature. This problem is known as the backward heat problem and is severely ill-posed. We show that this problem can be converted into the first Fredholm integral equation, and an algorithm of inversion is given using the Tikhonov's regularization method. The Newton root-finding algorithm for obtaining the regularization parameter is presented. We also present numerical computations that verify the accuracy of our approximation.
\end{abstract}

AMS Subject Classification: 34A55

Key Words: backward heat conduction problem, inverse problem, regularization, integral equation, ill-posed

\section{Introduction}

Inverse heat conduction problems have many applications in various branches of science and engineering,mechanical and chemical engineers, mathematicians and specialists in many other sciences branches are interested in inverse problems (see $[1,2,3,4,5]$ ), each with different application in mind. The main difficulty in the treatment of inverse problems is the instability of their solution in the presence of noise in the observed (measured) data, that is, the ill-posed nature

Received: September 11, 2012

(C) 2013 Academic Publications, Ltd.

$\S$ Correspondence author url: www.acadpubl.eu 
of the problem in the sense of hadamard. This problem not only defies easy solution, but has served to discourage the type of massive study that has accompanied direct or well-posed problems, see [6,7]. One could generically classify inverse problems into three types (all of them based on observations of the evolution of the involved physical system): identification of physical parameters or parameter identification; determination of the initial state of the system and determination of the boundary conditions, see $[8,9,10,11,12,13,14,15]$.

In this article we will examine some inversion techniques in order to estimate the initial temperature distribution of an inverse heat conduction problem .We show that this problem can be converted into the first Fredholm integral equation and an algorithm of inversion is given using the Tikhonov' s regularization method with Newton's method for the choice of the regularization parameter. Numerical experiments are presented to show the efficiency of the proposed method.

\section{Mathematical Model}

\subsection{The Direct Problem}

The direct (forward) problem consists of a transient heat conduction problem in a slab with adiabatic boundary condition and initially at a temperature denoted by $f(x)$

The mathematical formulation of this problem is given by the following heat equation:

$$
\begin{array}{cc}
\frac{\partial u}{\partial t}=D \frac{\partial^{2} u}{\partial x^{2}} & 0<x<L, t>0 \\
u(0, t)=0 & t>0 \\
u(L, t)=0 & t>0 \\
u(x, 0)=f(x) & 0 \leq x \leq L
\end{array}
$$

where $u(x, t)$ (temperature), $f(x)$ (initial condition), $x$ (spatial variable) and $t$ (time variable) are dimensionless quantities, $D$ denote the dispersion coefficient.

For the direct problem where the initial condition $f(x)$ is specified, the problem given by equation (1-4) is concerned with the determination of the temperature distribution $u(x, t)$ in the interior region of the solid as a function of time and position. 


\subsection{Inverse Problem}

For the inverse problem, the initial condition $f(x)$ is regarded as being unknown. In addition, an overspecified condition is also considered available. To estimate the unknown coefficient $f(x)$, the additional information

$$
u(x, T)=g(x)
$$

are given at time $T$, over a specified space interval $0 \leq x \leq L$. We note that the measured overspecified condition $u(x, T)=g(x)$ should contain measurement errors. Therefore the inverse problem can be stated as follows: by utilizing the above mentioned measured data, estimate the unknown function $f(x)$.

\section{Algorithm Analysis}

The solution of the direct problem for a given initial condition $f(x)$ is explicitly obtained using separation of variables, for $0<x<L, t \geq 0$

$$
u(x, t)=\int_{0}^{L} K(x, y, t) f(y) d y,
$$

where

$$
K(x, y, t)=\frac{2}{L} \sum_{n=1}^{\infty} e^{-\frac{(n \pi)^{2} D t}{L^{2}}} \sin \left(\frac{n \pi x}{L}\right) \sin \left(\frac{n \pi y}{L}\right)
$$

is an infinite series. Numerically, we can't handle infinite sums. Limit the sum to a finite number of expansion terms 100 which guarantees the convergence of the series. So

$$
K(x, y, t) \approx \frac{2}{L} \sum_{n=1}^{100} e^{-\frac{(n \pi)^{2} D t}{L^{2}}} \sin \left(\frac{n \pi x}{L}\right) \sin \left(\frac{n \pi y}{L}\right)
$$

Thus initial inverse problem is reduced to solving integral equation of the first kind.

$$
u(x, T)=g(x)=\int_{0}^{L} K(x, y, T) f(y) d y .
$$

The first step in the numerical treatment used in this research consists in discretiztion of equation (6) by the quadrature formula. The interval $[0, L]$ can be subdivided into equal intervals of width $h=\Delta y=\frac{L}{N}$. Let $y_{0}=0, y_{j}=j \Delta y$ and since the variable is either y or $\mathrm{x}$, let $x_{0}=y_{0}=0, x_{N}=y_{N}=L, x_{i}=$ 
$i \Delta y$, and (i.e. $\left.x_{i}=y_{i}\right)$. Also denote $f\left(x_{i}\right)$ as $f_{i}, g\left(x_{i}\right)$ as $g_{i}$, and $K\left(x_{i}, y_{i}, T\right)$ as $K_{i j}$. Now if the trapezoid rule is used to approximate the given equation, then:

$$
\begin{aligned}
g(x)= & \int_{0}^{L} K(x, y, T) f(y) d y \\
\approx & \Delta y\left[\frac{1}{2} K\left(x, y_{0}, T\right) f\left(y_{0}\right)+K\left(x, y_{1}, T\right) f\left(y_{1}\right)+\cdots\right. \\
& \left.+K\left(x, y_{N-1}, T\right) f\left(y_{N-1}\right)+\frac{1}{2} K\left(x, y_{N}, T\right) f\left(y_{N}\right)\right]
\end{aligned}
$$

or, more tersely:

$$
\begin{aligned}
g(x) \approx & \Delta y\left[\frac{1}{2} K\left(x, y_{0}, T\right) f_{0}+K\left(x, y_{1}, T\right) f_{1}+\cdots\right. \\
& \left.+K\left(x, y_{N-1}, T\right) f_{N-1}+\frac{1}{2} K\left(x, y_{N}, T\right) f_{N}\right]
\end{aligned}
$$

There are $N+1$ values of $f_{i}$, as $i=0,1,2 \cdots N$.Therefore the equation becomes a set of $N+1$ equations in $f_{i}$ :

$$
g_{i}=\Delta y\left[\frac{1}{2} K_{i 0} f_{0}+K_{i 1} f_{1}+\cdots K_{i(N-1)} f_{N-1}+\frac{1}{2} K_{i N} f_{N}\right], i=0,1,2 \cdots N .
$$

that give the approximate solution to $f\left(x_{i}\right)$ at $x=x_{i}$. This may also be written in matrix form:

$$
K F=G,
$$

where $\mathrm{K}$ is the matrix of coefficients

$$
K=\Delta y \cdot\left[\begin{array}{llll}
\frac{1}{2} K\left(x_{0}, y_{0}, T\right) & K\left(x_{0}, y_{1}, T\right) & \ldots & \frac{1}{2} K\left(x_{0}, y_{N}, T\right) \\
\frac{1}{2} K\left(x_{1}, y_{0}, T\right) & K\left(x_{1}, y_{1}, T\right) & \ldots & \frac{1}{2} K\left(x_{1}, y_{N}, T\right) \\
\ldots & \ldots & \ldots & \ldots \\
\frac{1}{2} K\left(x_{N}, y_{0}, T\right) & K\left(x_{N}, y_{1}, T\right) & \ldots & \frac{1}{2} K\left(x_{N}, y_{N}, T\right)
\end{array}\right]
$$

$F$ is the matrix of solutions

$$
F=\left[f\left(y_{0}\right), \cdots, f\left(y_{i}\right), \cdots, f\left(y_{N}\right)\right]^{T}
$$

and $G$ is the matrix of the nonhomogeneous part

$$
G=\left[g\left(x_{0}\right), \cdots, g\left(x_{i}\right), \cdots, g\left(x_{N}\right)\right]^{T} .
$$

The problem (7) is ill-posed in the sense that the inverse operator $A^{-1}$ of $A$ exists but it is not continuous. Hence, although the problem (7) has a unique 
solution, solving it directly will not give a right solution. Indeed, the linear operator A is so badly conditioned that any numerical attempt to directly solve (7) may fail.

In this note we describe Tikhonov regularization for finding a stable approximate solution to a linear ill-posed problem represented in the form of an operator equation:

$$
K F=G
$$

where instead of the exact data $G$, noisy $\operatorname{data} G_{\delta}$ is available with:

$$
\left\|G-G_{\delta}\right\| \leq \delta .
$$

In order to find a solution in stable manner, Tikhonov proposed to solve:

$$
F_{\alpha, \delta}=\underset{F \in R^{N+1}}{\arg \min } J_{\alpha}(F)=\left(\left\|K F-G_{\delta}\right\|_{2}^{2}+\alpha\|F\|_{2}^{2}\right),
$$

where the regularization parameter is found such that:

$$
\left\|K F_{\alpha, \delta}-G_{\delta}\right\|_{2}=\delta .
$$

The computation of the approximate solution $F_{\alpha, \delta}$ consists in solving the Euler equation corresponding to the functional $J_{\alpha}$. This equation has the form:

$$
\left(K^{*} K+\alpha I\right) F_{\alpha, \delta}=K^{*} G_{\delta},
$$

where $K^{*}$ is the adjoint operator of $K$ and $I$ is the identity operator.

The regularization parameter $\alpha$ satisfying the condition (8) and thus the associated solution $F_{\alpha, \delta}$ is typically determined by the Morozovs discrepancy principle $[16,17]$. How to solve the equation $\left\|K F_{\alpha, \delta}-G_{\delta}\right\|_{2}=\delta$, we introduce:

$$
\phi(\alpha)=\left\|K F_{\alpha, \delta}-G_{\delta}\right\|_{2}^{2}-\delta^{2} .
$$

Then the equation $\phi(\alpha)=0$ is equivalent to $\left\|K F_{\alpha, \delta}-G_{\delta}\right\|_{2}=\delta$, and it can be solved numerically for example, by Newton's method:

$$
\alpha_{j+1}=\alpha_{j}-\frac{\phi\left(\alpha_{j}\right)}{\phi^{\prime}\left(\alpha_{j}\right)} \quad j=0,1,2, \cdots .
$$

The derivative $\phi^{\prime}(\alpha)$ can be calculated as follows:

$$
\begin{aligned}
& \phi^{\prime}(\alpha)=\left[\left\langle K F_{\alpha, \delta}-G_{\delta}, K F_{\alpha, \delta}-G_{\delta}\right\rangle-\delta^{2}\right]_{\alpha}^{\prime} \\
& =\left\langle K \frac{d F_{\alpha, \delta}}{d \alpha}, K F_{\alpha, \delta}-G_{\delta}\right\rangle+\left\langle K F_{\alpha, \delta}-G_{\delta}, K \frac{d F_{\alpha, \delta}}{d \alpha}\right\rangle \\
& =2 \operatorname{Re}\left\langle K \frac{d F_{\alpha, \delta}}{d \alpha}, K F_{\alpha, \delta}-G_{\delta}\right\rangle
\end{aligned}
$$


We now compute $\frac{d F_{\alpha, \delta}}{d \alpha}$ by differentiating the identity:

$$
\alpha F_{\alpha, \delta}+K^{*} K F_{\alpha, \delta}=K^{*} G_{\delta}
$$

implicitly. Since the right-hand side does not depend on $\alpha$, one has:

$$
F_{\alpha, \delta}+\alpha \frac{d F_{\alpha, \delta}}{d \alpha}+K^{*} K \frac{d F_{\alpha, \delta}}{d \alpha}=0
$$

Solving for $\frac{d F_{\alpha, \delta}}{d \alpha}$ we get:

$$
\frac{d F_{\alpha, \delta}}{d \alpha}=-\left[\alpha I+K^{*} K\right]^{-1} F_{\alpha, \delta}=-\left[\alpha I+K^{*} K\right]^{-1}\left[\alpha I+K^{*} K\right]^{-1} K^{*} G_{\delta}
$$

Substituting this into $\phi^{\prime}(\alpha)$, one derives

$$
\begin{aligned}
& \phi^{\prime}(\alpha)=2 \operatorname{Re}\left\langle-K\left[\alpha I+K^{*} K\right]^{-1} F_{\alpha, \delta}, K F_{\alpha, \delta}-G_{\delta}\right\rangle \\
& =2 \operatorname{Re}\left\langle-K\left[\alpha I+K^{*} K\right]^{-1}\left[\alpha I+K^{*} K\right]^{-1} K^{*} G_{\delta}, K\left[\alpha I+K^{*} K\right]^{-1} K^{*} G_{\delta}-G_{\delta}\right\rangle
\end{aligned}
$$

\section{Numerical Examples}

In this section, we are going to demonstrate some numerical results for determining $f(x)$ in the inverse problem (1)-(5).

We use the $L_{\infty}$ error norm and the relative error to measure the difference between the numerical and analytical solutions. The $L_{\infty}$ error norm is defined by:

$$
L_{\infty}=\max _{0 \leq j \leq N}\left|f\left(x_{j}\right)-\tilde{f}\left(x_{j}\right)\right|
$$

and the relative error $(\mathrm{RE})$ is defined by:

$$
\mathrm{RE}=\sqrt{\frac{\sum_{j=0}^{N}\left|f\left(x_{j}\right)-\tilde{f}\left(x_{j}\right)\right|^{2}}{\sum_{j=0}^{N}\left|f\left(x_{j}\right)\right|^{2}}}
$$

where $x_{j}$ are test points and $N$ is the total number of uniformly distributed points on $[0,1] . f(x)$ is the exact solution and $\tilde{f}(x)$ is the numerical solution.

In our computations, we always take $N=40$. The noisy data $\left\{g^{\delta}\left(x_{j}\right)\right\} \mid \begin{aligned} & N=0 \\ & j=0\end{aligned}$ were assumed to contain some random errors. However, in practical applications, the reduplicated measurements are fairly difficult and even are impossible. 
Hence, in this paper, we consider the deterministic errors. Assume the observed data has the following noised form:

$$
g^{\delta}\left(x_{j}\right)=g\left(x_{j}\right)+\delta \sin \left(10 \pi x_{j}\right), \quad j=0,1,2, \cdots N .
$$

Example 1. In this example let us consider the following inverse problem:

$$
\begin{array}{lc}
\frac{\partial u}{\partial t}=\frac{\partial^{2} u}{\partial x^{2}} & 0<x<1, t>0 \\
u(0, t)=0 & t \geq 0 \\
u(1, t)=0 & t \geq 0 \\
u(x, 0)=f(x) & 0 \leq x \leq 1
\end{array}
$$

the over specified condition:

$$
u(x, 1)=g(x)=e^{-\pi^{2}} \sin (\pi x)
$$

The analytical solution of this example is $u(x, t)=e^{-\pi^{2} t} \sin (\pi x)$ and

$$
f(x)=\sin (\pi x)
$$

The regularization parameter $\alpha$ is chosen using Newton's method, the $L_{\infty}$ error norm and relative error RE are presented in Table 1. Also, the corresponding errors between the analytical and the estimated functions $f(x)$ in $x_{j}=0.1 j$ when $\delta=0.1$ are listed in Table 2 .

\begin{tabular}{lccc}
\hline$\delta$ & $\alpha$ & $L_{\infty}$ & $\mathrm{RE}$ \\
\hline 0.1 & $5.73185 \times 10^{-14}$ & $2.14202 \times 10^{-5}$ & $2.14247 \times 10^{-5}$ \\
0.05 & $8.72765 \times 10^{-15}$ & $3.26344 \times 10^{-6}$ & $3.26231 \times 10^{-6}$ \\
0.01 & $2.54233 \times 10^{-15}$ & $9.53403 \times 10^{-7}$ & $9.50310 \times 10^{-7}$ \\
0.001 & $9.81035 \times 10^{-16}$ & $3.69100 \times 10^{-7}$ & $3.66705 \times 10^{-7}$ \\
\hline
\end{tabular}

Table 1: The $L_{\infty}$ and the relative errors for $f(x)$, with $\delta=$ $0.1,0.05,0.01,0.001$

Example 2. In this example let us consider the following inverse problem

$$
\begin{array}{lc}
\frac{\partial u}{\partial t}=D \frac{\partial^{2} u}{\partial x^{2}} & 0<x<1, t>0 \\
u(0, t)=0 & t>0 \\
u(1, t)=0 & t>0 \\
u(x, 0)=f(x) & 0 \leq x \leq 1
\end{array}
$$




\begin{tabular}{lccc}
\hline$j$ & Exact $f\left(x_{j}\right)$ & Numerical $\tilde{f}\left(x_{j}\right)$ & $f\left(x_{j}\right)-\tilde{f}\left(x_{j}\right)$ \\
\hline 1 & 0.309016994374947 & 0.309016994374947 & $6.621267118533 \times 10^{-6}$ \\
2 & 0.587785252292473 & 0.587772658514349 & $1.259377812457 \times 10^{-5}$ \\
3 & 0.809016994374947 & 0.808999662566453 & $1.733180849472 \times 10^{-5}$ \\
4 & 0.951056516295154 & 0.951036138131767 & $2.037816338640 \times 10^{-5}$ \\
5 & 1 & 0.999978579729737 & $2.142027026342 \times 10^{-5}$ \\
6 & 0.951056516295154 & 0.951036139325882 & $2.037696927137 \times 10^{-5}$ \\
7 & 0.809016994374947 & 0.80899966217245 & $1.733220249233 \times 10^{-5}$ \\
8 & 0.587785252292473 & 0.587772660016492 & $1.259227598149 \times 10^{-5}$ \\
9 & 0.309016994374948 & 0.309010373872585 & $6.620502362109 \times 10^{-6}$ \\
10 & $1.22464679914735 \times 10^{-16}$ & $6.12310280754787 \times 10^{-17}$ & $6.123365183926 \times 10^{-17}$ \\
\hline
\end{tabular}

Table 2: The analytical and numerical results for the $f\left(x_{j}\right)$ in $x_{j}=0.1 j$ where $\delta=0.1$

the initial condition

$$
f(x)= \begin{cases}2 x & 0 \leq x \leq 0.5 \\ 2(1-x) & 0.5 \leq x \leq 1\end{cases}
$$

where $D=0.01$, the exact solution is given by using the separation of variables

$$
u(x, t)=\sum_{n=0}^{\infty} \frac{8}{\pi^{2}(2 n+1)^{2}} \cos \left(\frac{(2 n+1) \pi(2 x-1)}{2}\right) e^{\left[-D \pi^{2}(2 n+1)^{2} t\right]}
$$

The experimental data $u(x, T)=g(x)$ (measured temperatures at $T=1$ ) is obtained from the exact solution by taking the sum of the first one hundred terms. The $L_{\infty}$ error norm and relative error $R E$ are presented in Table 3, Also, the corresponding errors between the analytical and the estimated functions $f(x)$ in $x_{j}=0.1 j$ when $\delta=0.1$ are listed in Table 4.

\begin{tabular}{lccc}
\hline$\delta$ & $\alpha$ & $L_{\infty}$ & $\mathrm{RE}$ \\
\hline 0.1 & 0.00036282 & 0.0634335 & 0.0312757 \\
0.05 & 0.00021836 & 0.0611471 & 0.0287479 \\
0.01 & 0.00005996 & 0.0547497 & 0.0237954 \\
0.001 & 0.00001008 & 0.0478144 & 0.0203349 \\
\hline
\end{tabular}

Table 3: The $L_{\infty}$ and the relative errors for $f(x)$, with $\delta=$ $0.1,0.05,0.01,0.001$

The graph of the analytical and the estimated functions for $f(x)$ is given in Figure 5-8

From the previous two numerical examples, it can be seen that the numerical results are quite satisfactory. Even with the noise level of input data up to 


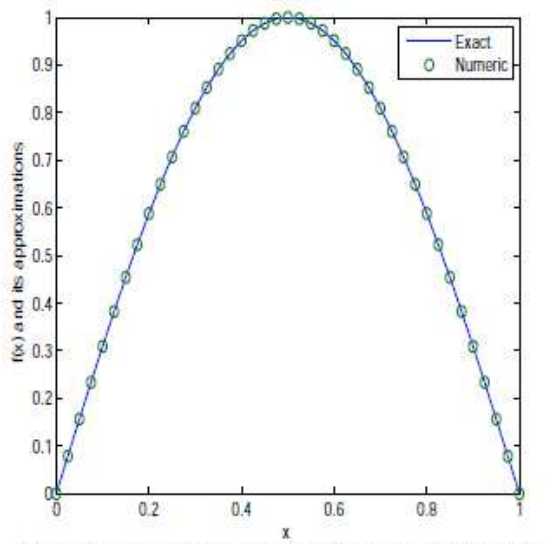

Fig 1 Comparison between the exact resuts and the present numerical resuls, $\delta=0.1$

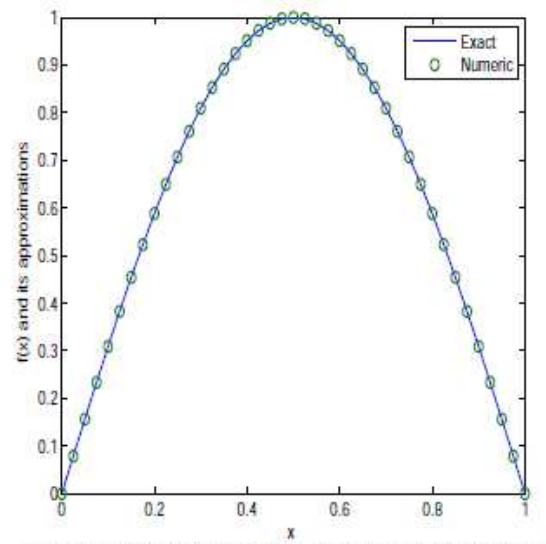

Fig. 2 Comparison between the exact resuts and the present numerical resuls, $\delta=0.05$

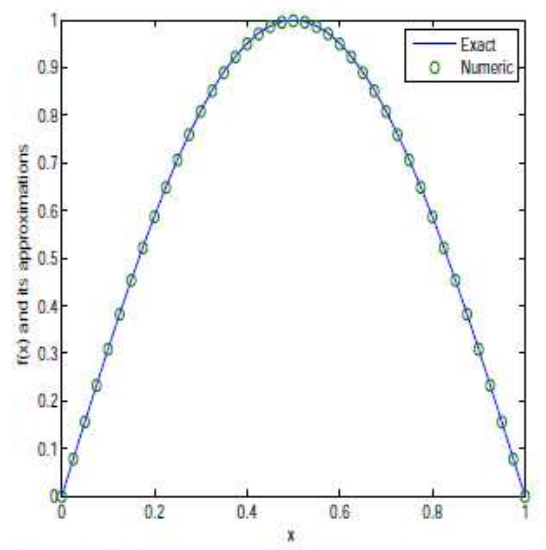

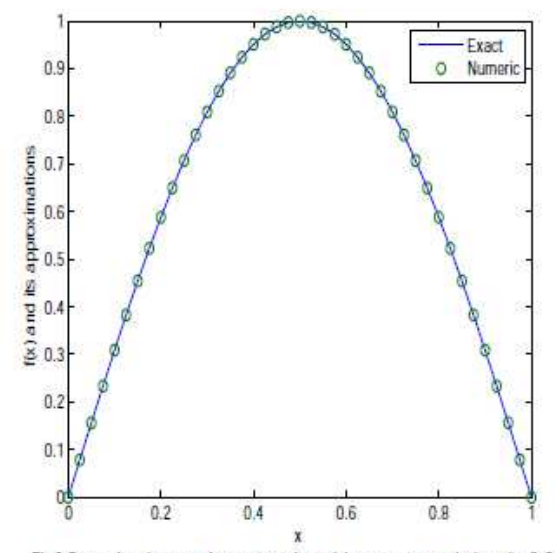

Fig 4 Comparison between the exact results and the presert numerical resuts, $\delta=0.001$

Figure 1: Figure 1-4 show the comparison between exact and numerical solutions of $f(x)$ with noise $\delta=0.1,0.05,0.01,0.001$, respectively, for Example 1.

$\delta=0.1$, the numerical solutions are still in good agreement with the exact solutions 


\begin{tabular}{lccc}
\hline$j$ & Exact $f\left(x_{j}\right)$ & Numerical $\tilde{f}\left(x_{j}\right)$ & $f\left(x_{j}\right)-\tilde{f}\left(x_{j}\right)$ \\
\hline 1 & 0.2 & 0.203285479287456 & 0.0032854792874559 \\
2 & 0.4 & 0.399171118110754 & 0.0008288818892464 \\
3 & 0.6 & 0.590641061060895 & 0.0093589389391055 \\
4 & 0.8 & 0.818696676546333 & 0.0186966765463328 \\
5 & 1 & 0.945250250199793 & 0.0547497498002067 \\
6 & 0.8 & 0.818696676542829 & 0.0186966765428294 \\
7 & 0.6 & 0.590641061065385 & 0.0093589389346149 \\
8 & 0.4 & 0.399171118106812 & 0.0008288818931877 \\
9 & 0.2 & 0.203285479288568 & 0.0032854792885684 \\
10 & 0 & $3.39804782379576 \times 10^{-17}$ & $3.39804782379576 \times 10^{-17}$ \\
\hline
\end{tabular}

Table 4: The analytical and numerical results for the $f\left(x_{j}\right)$ in $x_{j}=0.1 j$ where $\delta=0.1$

\section{Conclusion}

This paper deals with the effective algorithms for solving the backward heat problem and the following results are obtained.

1. The present study, successfully applies the numerical method involving the Tikhonov's regularization method in conjunction with the first Fredholm integral equation to the inverse heat conduction problems.

2. From the illustrated example it can be seen that the proposed numerical method is efficient and accurate to estimate the initial condition $f(x)$.

\section{Acknowledgments}

The work of the author is supported by the Special Funds of the National Natural Science Foundation of China (Nos. 51179151, 51190093). The author would like to thank the referees for constructive suggestions and comments.

\section{References}

[1] A. Shidfar, G.R. Karamali, J. Damirchi, An inverse heat conduction problem with a nonlinear source term, Nonlinear Analysis, Theory Meth. Appl., 65 (2006), 615-621. 

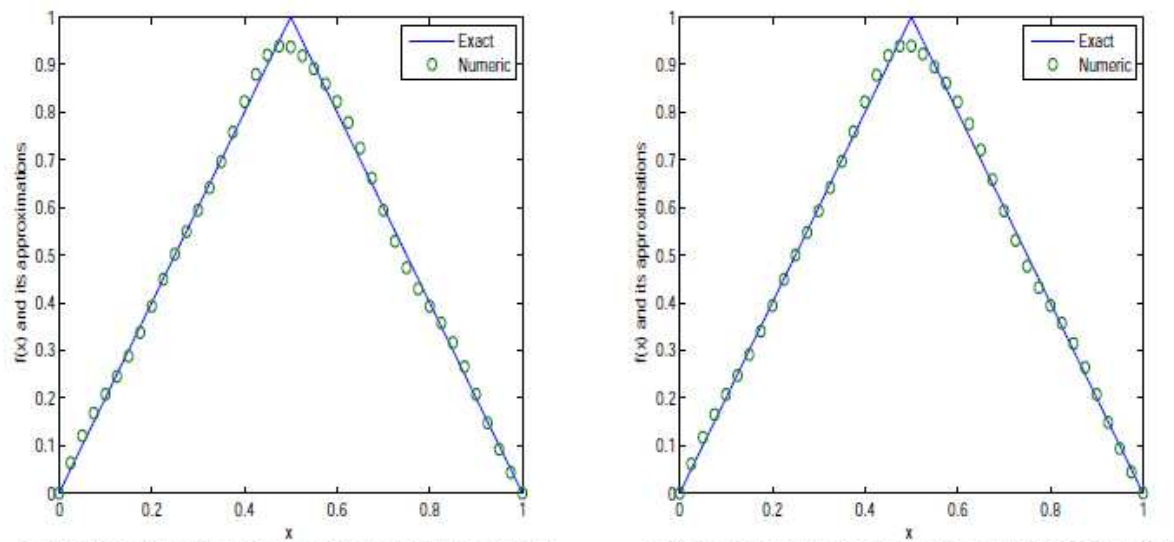

Fig. 5 Comparison between the exact resuts and the present numerical resuts, $\delta=0.1$
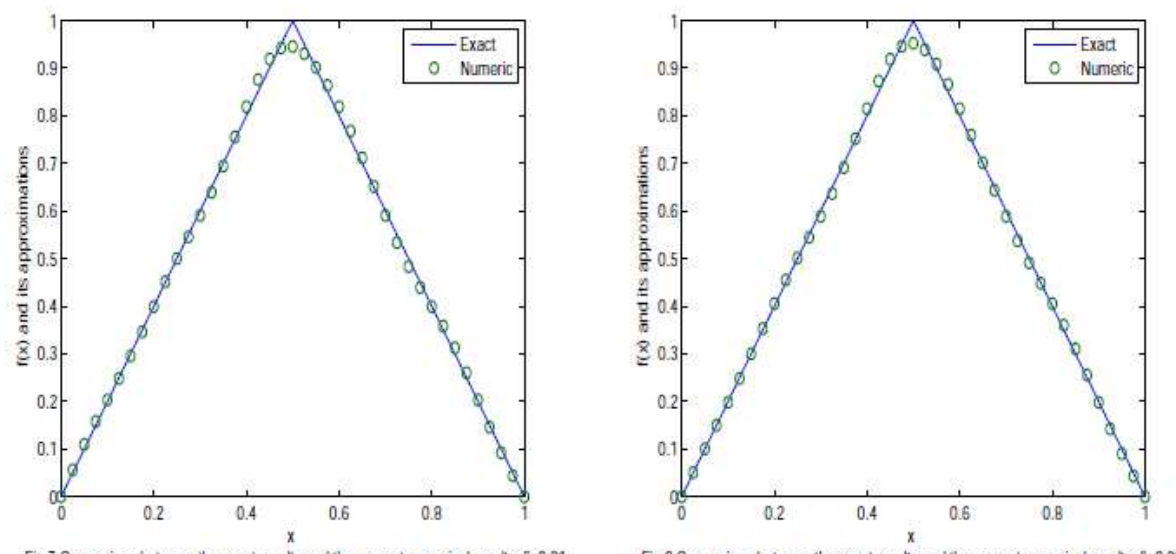

Fig.7 Comparison betwean the exsct results and the presert rumerical resuts, $0=0.01$

Fig. 8 Comparison between the exact results and the present numerical resuts, $8=0.001$

Figure 2: Figure 5-8 show the comparison between exact and numerical solutions of $f(x)$ with noise $\delta=0.1,0.05,0.01,0.001$, respectively, for Example 2.

[2] R. Pourgholi, N. Azizi, Y.S. Gasimov, F. Aliev, H.K. Khala, Removal of numerical instability in the solution of an inverse heat conduction problem, Communications in Nonlinear Science and Numerical Simulation, 14, No. 6 (2009), 2664-2669.

[3] N. Azizi, A. Shidfar, R. Pourgholi, A stable solution for an inverse heat conduction problem, IUST International Journal of Engineering Science, 
16, No. 32005 .

[4] J. Shi, J. Wang, Inverse problem of estimating space and time dependent hot surface heat flux in transient transpiration cooling process, International Journal of Thermal Sciences, 48 (2009), 1398-1404.

[5] B.T. Johansson, D. Lesnic, A procedure for determining a spacewise dependent heat source and the initial temperature, Applicable Analysis, 87, No. 3 (2008), 265-276.

[6] A. Hasanov, P.C. Duchateau, B. Pektas, Inverse and ill-posed problems, 14, No. 5 (2006), 435-463.

[7] D.D. Trong, N.H. Tuan, Regularization and error estimates for nonhomogeneous backward heat problems, Electron. J. Diff. Eqns., 2006, No. 04 (2006), 1-10.

[8] M. Ebrahimian, R. Pourgholi, M. Emamjome, P. Reihani, A numerical solution of an inverse parabolic problem with unknown boundary conditions, Applied Mathematics and Computation, 189, No. 1 (2007), 228-234.

[9] M. Dehghan, Determination of an unknown parameter in a semi-linear parabolic equation, Math Problems Eng., 8, No. 2 (2002), 111-22.

[10] W.B. Muniz, A comparison of some inverse methods for estimating the initial condition of the heat equation, J. Comput. Appl. Math., 103 (1999), 145-163.

[11] N.S. Mera, L. Elliott, D.B. Ingham, An inversion method with decreasing regularization for the backward heat conduction problem, Numer. Heat Transfer B, 42 (2002), 215-230.

[12] C.-S. Liu, Group preserving scheme for backward heat conduction problems, Int. J. Heat Mass Transfer, 47 (2004), 2567-2576.

[13] C.-S. Liu, A highly accurate LGSM for severely ill-posed BHCP under a large noise on the final time data, Int. J. Heat Mass Transfer, 53 (2010), 4132-4140.

[14] B.T. Johansson, D. Lesnic, A method of fundamental solutions for transient heat conduction, Eng. Anal. Bound. Elem., 32 (2008), 697-703. 
[15] R. Model, U.Hammerschmidt: Numerical methods for the determination of thermal properties by means of transient measurements, Advanced Computational Methods in Heat Transfer, WIT Press, No. 1 (2000), 407-416.

[16] V.A. Morozov, Choice of a parameter for the solution of functional equations by the regularization method, Sov. Math. Doklady, 8 (1967), 10001003.

[17] V.A. Morozov, Methods for Solving Incorrectly Posed Problems, Springer Verlag Berlin (1984). 
